MRS Advances (C) 2017 Materials Research Society

DOI: 10.1557/adv.2017.81

\title{
Micro/Nanoscale Tribological and Mechanical Investigation of the Articular Surfaces of Katydid Leg Joints: Potential for the Novel Bioinspired Lubrication Systems
}

Jun Kyun $\mathrm{Oh}^{1}$, Cengiz Yegin ${ }^{1}$, and Mustafa Akbulut ${ }^{1,2}$

${ }^{1}$ Department of Materials Science and Engineering, Texas A\&M University, College Station, TX 77843, U.S.A.

${ }^{2}$ Artie McFerrin Department of Chemical Engineering, Texas A\&M University, College Station, TX 77843, U.S.A.

\begin{abstract}
Insects are recognized with their ability to efficiently move, operate, and function, and hence are inspiration for the design of micromechanical systems. This work deals with the structural, mechanical, and frictional characterization of the leg joint articulations of the katydid (Orthoptera: Tettigoniidae). For the katydids, the tibia joints were found to show a nanosmooth texture while the femur joint had a micro/nanotextured surface characteristics. The nanotexture was a two-tone periodic patterns with the hierarchical structures involving cylindrical ridges that are covered with nanoscale lamellar patterns perpendicular to the long axis and valleys between ridges that are decorated with the hillock patterns. The tibia and femur contact regions showed the reduced elastic modulus $\left(E_{\mathrm{r}}\right)$ values ranging from $0.88 \pm 0.01 \mathrm{GPa}$ to $3.90 \pm 0.11 \mathrm{GPa}$. The friction coefficient $(\mu)$ value of $0.053 \pm 0.001$ was recorded for the sliding contact of the tibia joint against the femur joint in air under dry conditions. The low friction values are attributed to the reduced real area of contact between the joint pair due to the coupling of the nanosmooth surfaces against the hierarchically nanotextured surfaces.
\end{abstract}

\section{INTRODUCTION}

Through the evolution of millions of years to thousands of years, the natural species have developed optimized multifunctional composite materials and geometric structures to achieve efficient functions and operations required in their lifespan [1,2]. Biotribology is one of the fastest-growing research areas of the science and engineering dealing with all aspects of tribology derived from the biological systems [3]. Studying materials based on the biological systems is to offer useful clues and design motifs for developing new materials for various engineering applications [4,5]. Biomimetic materials are of great interest in the field of tribology, which seeks to develop novel lubrication systems and coating for enhanced efficiency and durability [6].

In this work, we have investigated the articular surfaces of the katydid joints and gained some insights into why insect legs function durably and effectively. The results from the fundamental analysis and characterization showed that the tibial and femoral articular surfaces indeed exhibit the promising tribological and mechanical properties due to very sophisticated hierarchical structures. The investigation was carried out by high resolution imaging and 
mechanical analysis techniques using scanning electron microscopy (SEM), nanotribometry, and nanoindentation. The resulting tribological and mechanical comprehension have significant potential to expand current knowledge of designing the tribological materials as well as to give rise to the bioinspired strategies for fabricating material surfaces with the superior tribological behavior. Finding the unique natural surfaces is an attempt to draw out some of the potential for the biomimetic-based design and encourage for further advanced research in the lubrication systems.

\section{EXPERIMENTAL DETAILS}

\section{Sample preparation}

Live katydids (Orthoptera: Tettigoniidae) were obtained from the Department of Entomology, Texas A\&M University, College Station, TX, U.S.A. The samples were prepared from the rear leg joint of the katydids. For experimental studies, fresh katydids were anesthetized with carbon dioxide. Surgical separation with a disposable scalpel and removal with an insect handling tweezer allows the cover plate to be extracted, leaving the thigh bone (femur) and shin bone (tibia) of the leg joint. To avoid desiccation of specimen, fresh leg joint was tested immediately after dissecting from the body.

\section{Structural characterization of insect joints}

Photographs were obtained using a video camera (HDR-XR160; Sony Corp., Tokyo, Japan). The specimens were examined by scanning electron microscope (SEM, JSM-7500F; JEOL, Tokyo, Japan). To minimize possible charging effects, the samples were coated with 15 $\mathrm{nm}$ of platinum/palladium (Pt/Pd) prior to SEM imaging. The SEM was operated at an emission current of $20 \mu \mathrm{A}$ and an accelerating voltage of $1 \mathrm{kV}$. Insect features such as width, spacing, and periodicity were determined from SEM imaging of the top view and side view.

\section{Friction force and wear measurements}

The friction response was conducted via nanotribological testing using a nanotribometer (Anton Paar TriTec SA, Peseux, Switzerland). All tests were conducted using a cantilever spring with the tangential and normal stiffness of $36.8 \mathrm{~N} / \mathrm{m}$ and $55.9 \mathrm{~N} / \mathrm{m}$, respectively, at the normal loads of $1,3,5,7$, and $10 \mathrm{mN}$. To ensure a sliding geometry similar to natural orientation and arrangement of insect joints, the tibia was mounted on an angled holder using the instant glue while the femur was glued on the base stage. The analyses were carried out under ambient conditions (i.e., temperature of $23{ }^{\circ} \mathrm{C}$ and relative humidity of $65 \%$ ) in the absence of any lubricant. The mechanical robustness was investigated via nanotribological testing using a nanotribometer. The contact region of the tibia was sheared on the contact region of the femur for 100 cycles at the normal load of $15 \mathrm{mN}$. 

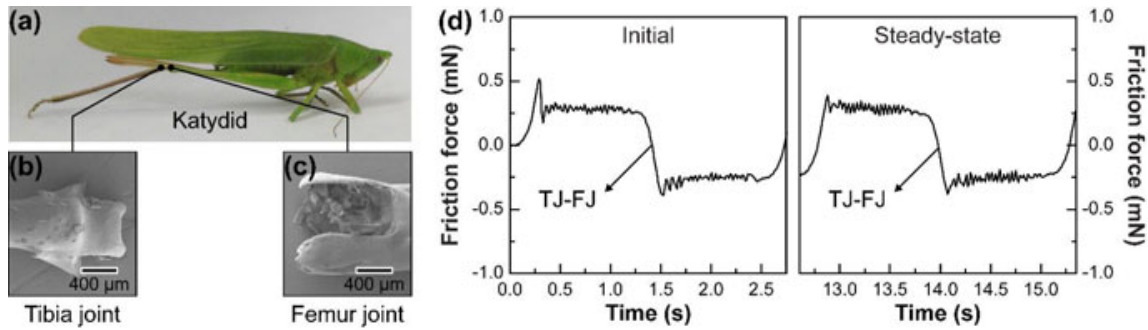

Figure 1. (a) The photograph of the katydid. SEM micrographs of its (b) tibia joint and (c) femur joint. (d) The representative initial and steady-state friction traces resulting from the sliding of tibia joint (TJ)-femur joint (FJ) pair. Steady-state friction decreased from initial friction by $\sim 2.3 \%$ at a sliding speed of $0.5 \mathrm{~mm} / \mathrm{s}$.

\section{$\underline{\text { Nanoindentation measurements }}$}

In order to measure mechanical properties, a nanoindenter (Ubi 1; Hystiron Inc., Eden Prairie, MN, U.S.A.) was used by positioning the indenter tip at the area of interest. While an indenter tip was brought into contact with the sample surface, the applied load and penetration depth (caused by the displacement) into the specimens were recorded simultaneously. The load profile consisted of three $10 \mathrm{~s}$ intervals: a linearly increase from zero to a maximum load, the maximum being held, and a linear decrease back to zero. Different maximum loads ranging from $100 \mu \mathrm{N}$ to $4000 \mu \mathrm{N}$ were applied to penetrate the joints over a range of depths. The analysis was based on the simple assumptions that unloading is fully reversible. The analyses were carried out under ambient conditions.

\section{RESULTS}

\section{Frictional properties of insect joints}

Friction traces provide information useful for assessing the processes and interactions of the two sliding surfaces $[7,8]$. The photographs and SEM micrographs of the surfaces studied are given in Figures 1a-c, which show that the katydids have saltatorial legs with an elongated tibia and femur adapted for jumping. Figure $1 \mathrm{~d}$ shows the representative traces obtained during shearing at the normal load of $5 \mathrm{mN}$. The analysis of which revealed the friction was accompanied by stick-slip fluctuations.

\section{$\underline{\text { Structural characterization and mechanical properties of insect joints }}$}

We investigated the surface topography of insect joints to gain insights into the observed tribological trends. SEM micrographs show that the joint part of the femoral articular surfaces exhibit two-tone periodic patterns with the hierarchical structures: (i) cylindrical ridges that are 

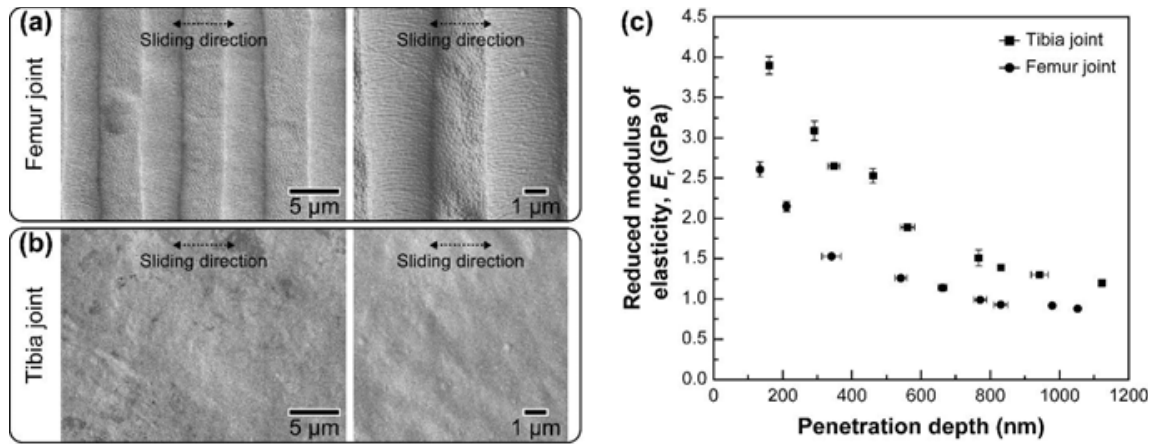

Figure 2. SEM micrographs showing the topographical details of the (a) femoral articular joint and (b) tibial articular joint. (c) The graph shows the reduced elastic modulus $\left(E_{\mathrm{r}}\right)$ versus penetration depth for the tibial and femoral articular surfaces of the katydid joints. The results are collected from the tibia contact regions and femur contact regions of the articular surfaces.

covered with nanoscale lamellar patterns perpendicular to the long axis and (ii) valleys that are separated by a certain distance and decorated with the hillock patterns (Figure 2a). The analysis of SEM micrographs revealed that the diameter of the ridge is $1.9 \pm 0.1 \mu \mathrm{m}$ and the ridge spacing is $1.7 \pm 0.2 \mu \mathrm{m}$. On the other hand, the tibial articular surfaces are relatively smooth and contain no well-defined textures (Figure $2 b$ ).

Furthermore, we measured the reduced elastic modulus $\left(E_{\mathrm{r}}\right)$ of the articular surfaces (Figure 2c). The reduced modulus of elasticity appeared to decrease with increasing penetration depth up to $\sim 700 \mathrm{~nm}$, then remain constant. The plateau value was $1.30 \pm 0.05 \mathrm{GPa}$ for tibia joints and $0.91 \pm 0.02 \mathrm{GPa}$ for femur joints.

\section{Wear behavior of insect joints}

Upon completing 100 sliding cycles at the normal load of $15 \mathrm{mN}$, the bare (Figure 3a) and sheared surfaces were probed with SEM to determine the resultant wear characteristics. No considerable change was detected on either of the tibia-femur pair, indicating a high wear resistance for this combination (Figure 3b).

\section{DISCUSSION}

Natural systems show possible solutions to provide ultra-low friction coatings and lubrication systems $[9,10]$. Previously, surface topography of the insect species were investigated, e.g., spherically capped, diamond-like shape, rod-shaped, interconnected netting, and curved [11]. Since the katydid joints show interesting nanotopographical features, friction behavior at the micro/nanoscale is important because macroscopic laws of friction do not 

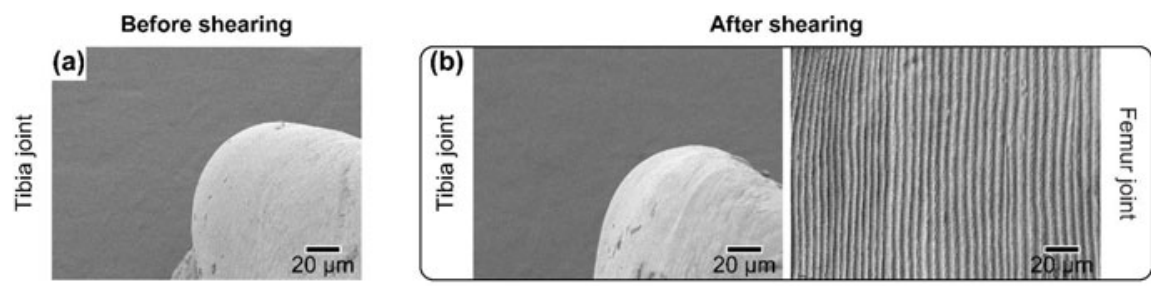

Figure 3. SEM micrograph of (a) tibia joint before shearing and (b) the combination of tibia joint (TJ)-femur joint (FJ) pair sliding contacts after 100 shearing cycles at the normal load of $15 \mathrm{mN}$ and room temperature under atmospheric conditions.

generally apply to micro/nanoscale materials [12]. As materials are shrinking in size to nanoscale, surface forces such as friction, wear, and adhesion become significantly critical. Insect joints show an exceptional mechanical durability in spite of the repetitive habits such as jumping, running, walking, swimming, digging, and grabbing. Due to their unique nanostructures and chemistries of the articulating surfaces, insect joints can be truly multifunctional hybrid materials. As a result, articular surfaces can provide low friction as well as wear resistance [13]. In summary, various factors which affect frictional behavior of a micro/nanotextured surface at the nanoscale can be discussed in detail. For example, density of texture, size of contact area, and shape of texturing structure [14].

\section{$\underline{\text { Structural characteristics of insect joints }}$}

In general, topographies of any mating surfaces strongly influence their tribological behavior [15]. The surfaces should be as smooth as possible to minimize the maximum contact stresses applied on the asperity level. However, for extremely smooth surfaces, the wear rates can be high, especially in micro/nanoscale systems, because the extremely smooth surfaces have very large real contact areas that increase the adhesion contribution of frictional forces and thereby can cause higher wear rates [16]. The nanoscale lamellar patterns which are perpendicular to the microscale groove patterns on the articular surfaces of the katydid joints can be attributed to reducing the sliding friction by minimizing the points of the contact area. The friction decreased by the lamellar patterns between the solid surfaces and air near the surfaces. The morphology of the lamellar patterns aligned perpendicular to the sliding direction can reduce the air resistance [17].

\section{$\underline{\text { Nanomechanical characteristics of insect joints }}$}

Nanoindentation results showed the stiffness of the articulation structure [18]. The elastic modulus is a crucial parameter in the study of shear and compression that will occur when the two surfaces come together into contact [19]. An important characteristic of most natural 
lubrication systems is that they usually involve relatively small elastic modulus values, as exemplified by the movements performed by biological entities such as slugs, eyes, and cartilage-coated articular joints [20].

\section{Characterization of wear behavior of insect joints}

Understanding the antiwear mechanisms of the biological material surfaces is an important topic in the field of biotribology [21]. Wear behavior of the nanoscale objects reduced with lower contact area [22]. Typically, wear is determined by the interplay of the two opposing properties (i.e., ductility and hardness). Wear can be reduced by modifying the surface morphology so that it acquires higher ductility, allowing greater plastic deformation. The soft biological material surfaces can reduce the wear due to the delamination, resulting in less net deformation [23].

\section{CONCLUSIONS}

In this paper, the tribological and mechanical properties of the tibial and femoral articular surfaces of the katydid joints, specialized for the jumping activities were investigated. We demonstrated that the katydid joints possess exceptional low frictional properties, presumably due to the micro/nanoscale periodic patterns (i.e., hillock, lamellar, and groove) with the hierarchical structures on the femoral articular surfaces which are in contact with the nanosmooth tibia surfaces. Our new finding can be used to further probe in the mechanisms of the friction to lead to the development of more effective lubrication systems and coatings through bioinspiration. Since the friction, wear, efficiency, durability, and long-term sustainability are the key contemporary issues in the fields of micro/nanoscale biomechanics and biomaterials science, our work has potential to advance the current state-of-the-art by determining the structural and morphological properties of the katydid joints that have not yet been studied.

\section{ACKNOWLEDGMENTS}

This project was supported by the National Science Foundation (NSF) under Grant Number: 1434421.

\section{REFERENCES}

1. U.G.K. Wegst, H. Bai, E. Saiz, A.P. Tomsia, R.O. Ritchie, Nat. Mater. 14, $23-36$ (2014).

2. B. Bhushan, Langmuir 28, 1698-1714 (2012).

3. Z.R. Zhou, Z.M. Jin, Biosurf. Biotribol. 1, 3-24 (2015).

4. J. Aizenberg, P. Fratzl, Adv. Funct. Mater. 23, 4398-4399 (2013).

5. B.A. Kheireddin, T.C. Williams, M. Akbulut, Tribol. Int. 50, 76-81 (2012).

6. J. Meng, P. Zhang, S. Wang, Chem. Soc. Rev. 45, 237-251 (2016). 
7. B. Gueye, Y. Zhang, Y. Wang, Y. Chen, Nano Lett. 15, 4704-4712 (2015).

8. C. Drummond, P. Richetti, "Surface Forces Apparatus in Nanotribology", Fundamentals of Friction and Wear on the Nanoscale, (Springer, 2015) pp. 17-34.

9. J. Lomakin, P.A. Huber, C. Eichler, Y. Arakane, K.J. Kramer, R.W. Beeman, M.R. Kanost, S.H. Gehrke, Biomacromolecules 12, 321-335 (2011).

10. C. Guschlbauer, H. Scharstein, A. Buschges, J. Exp. Biol. 210, 1092-1108 (2007).

11. G.S. Watson, J.A. Watson, S. Hu, C.L. Brown, B.W. Cribb, S. Myhra, Int. J. Nanomanuf. 5, 112-128 (2010).

12. Y. Mo, K.T. Turner, I. Szlufarska, Nature 457, 1116-1119 (2009).

13. A. Erdemir, Tribol. Int. 38, 249-256 (2005).

14. X. Zhang, J. Jia, Surf. Rev. Lett. 22, 1530001 (2015).

15. M.H. Müser, Phys. Rev. Lett. 100, 055504 (2008).

16. J. Israelachvili, N. Maeda, K.J. Rosenberg, M. Akbulut, J. Mater. Res. 20, 1952-1972 (2005).

17. C. Yu, H. Yu, G. Liu, W. Chen, B. He, Q.J. Wang, Tribol. Lett. 53, 145-156 (2014).

18. S. Enders, N. Barbakadse, S.N. Gorb, E. Arzt, J. Mater. Res. 19, 880-887 (2004).

19. E. Gerde, M. Marder, Nature 413, 285-288 (2001).

20. M. Urbakh, J. Klafter, D. Gourdon, J. Israelachvili, Nature 430, 525-528 (2004).

21. S. Amini, A. Miserez, Acta Biomater. 9, 7895-7907 (2013).

22. D. Maharaj, B. Bhushan, Friction, Mater. Sci. Eng. R-Rep. 95, 1-43 (2015).

23. H. Yao, M. Dao, T. Imholt, J. Huang, K. Wheeler, A. Bonilla, S. Suresh, C. Ortiz, Proc. Natl. Acad. Sci. 107, 987-992 (2010). 